\title{
Fluorinated waste and firefighting activities: biodegradation of hydrocarbons from petrochemical refinery soil co-contaminated with halogenated foams
}

\author{
Renato Nallin Montagnolli ${ }^{1} \cdot$ Paulo Renato Matos Lopes ${ }^{2} \cdot$ Ederio Dino Bidoia $^{1}$
}

Received: 16 November 2017 / Accepted: 18 February 2018 / Published online: 27 February 2018

(C) Springer-Verlag GmbH Germany, part of Springer Nature 2018

\begin{abstract}
Perfluorinated compounds, including fluorotelomers, are important constituents of firefighting foams to extinguish fuel fires in the petrochemical industry, airports, and at fire-training sites. In this study, we monitored the biodegradation process in a cocontamination scenario with monoaromatic hydrocarbons commonly found in fuels (benzene, toluene) and fluorotelomers. The $\mathrm{CO}_{2}$ production rates were evaluated by a factorial design taking into account the effect of seasonality at in situ natural attenuation processes. Headspace analysis by gas chromatography with a thermal conductivity detector (GC-TCD) was applied to detect $\mathrm{CO}_{2}$ production, whereas monoaromatics were analyzed by gas chromatography coupled to mass spectrometry (GC-MS). According to our results, seasonality had a detectable effect during summer, yielding different $\mathrm{CO}_{2}$ production rates. Higher temperatures increased $\mathrm{CO}_{2}$ production rate, while higher concentrations of fluorotelomer inhibited the biodegradation process. On average, benzene and toluene were depleted 17.5 days earlier in control assays without fluorotelomers. Toluene removal efficiency was also notably higher than benzene. The noticeable decrease in degradation rates of monoaromatics was caused by perfluorinated compounds that are possibly linked to metabolic inhibition mechanisms. Fluorotelomer diminished catabolism in all of our batch cultures. In addition to this, an alternative production of by-products could be detected. Thus, we propose that transient components of the benzene and toluene degradation may be differentially formed, causing the benzene, toluene, and perfluorinated cocontaminations to go through switched metabolic stages under the presence of fluoride in a contamination scenario.
\end{abstract}

Keywords Perfluorinated compounds · Fluorotelomer - Bioremediation · Chromatography · Halogenated waste · Bioremediation · Benzene $\cdot$ Toluene

\section{Introduction}

Petroleum derivatives can be hazardous contaminants, as many of its compounds persist in the environment for an extended amount of time. Fires and explosions are another, more severe, way that petroleum can be introduced to ecosystems. When fossil fuels reach the ground, most of their components are split into

Responsible editor: Robert Duran

Ederio Dino Bidoia

ederio@rc.unesp.br

1 Department of Biochemistry and Microbiology, Biosciences Institute, Sao Paulo State University (UNESP), Rio Claro, São Paulo, Brazil

2 College of Agricultural and Technological Sciences, São Paulo State University (UNESP), Dracena, São Paulo, Brazil three phases: dissolved, liquid, and gaseous. A minor fraction of this mixture is prone to dissolving in groundwater, whereas most of the portion is retained in the porous spaces between soil particles in its pure liquefied form (also known as residual saturation). A third portion evaporates, leading to atmospheric contamination (Nadim et al. 1999; Freije 2015; Tobiszewski and Namieśnik 2015; Mishra et al. 2016; Cholakov 2016; Zhuo et al. 2017). The petroleum contamination is relevant due to the potential to contaminate aquifers and springs that often supply urban and industrial demand (Qin et al. 2009; Choi and Lee 2011; Montagnolli et al. 2014; Postigo et al. 2017).

Many references (NFPA 1991; Chettouh et al. 2016; Zhang et al. 2016; Runefors et al. 2017) state that fires are prone to occur in both confined areas (such as fuel storage tanks at refineries or gas stations) and in wide-open facilities (such as wells and oil spills). Therefore, accidents are potential causes of catastrophic combustion of fuels. In this context, water is 
not the most appropriate substance to suppress fires, due to its ineffective extinguishing properties. Firefighting foams are the proper extinguishing resource. They are better flame retardants when hydrocarbons ignite. However, these foams also combine with soil particles on long-term, causing a mixed contamination situation along with the remaining nonignited hydrocarbon (Nolan 2011; Borg and Njå 2013). Therefore, petroleum pollution can be intensified by such firefighting foams, due to their formulation, which is mostly based on fluorinated substances.

Emergency situations related to firefighting are often encountered in the petroleum industry, as well as airports and even fire training sites at military bases. These possibly disastrous scenarios are all solved using aqueous film-forming foams, which are substances specifically designed to extinguish hydrocarbon fires. The main components of such foams are long carbon chains containing many strong $\mathrm{C}-\mathrm{F}$ bonds (perfluorinated chains) that act by reducing surface tension (Dauchy et al. 2017). Fluorotelomers are the active substance within firefighting foam formulations that cause improved spreading and fire suppression (Donaldson 2016). Certainly, firefighting occurs in many scenarios, but they are all linked to other petroleum-based substances such as diesel, kerosene, and other fuels, hence causing a much more complex co-contamination in the environment (Vecitis et al. 2009; Hinnant et al. 2017).

The co-contamination of hydrocarbons with firefighting foams has a considerable impact on biodegradation rates. Petroleum products, such as gasoline, also contain toxic monoaromatic compounds (e.g., benzene and toluene) as reported by Wang et al. (2016), Fernández et al. (2016), Meyer et al. (2014), and Oberoi and Philip (2017). Among hydrocarbon found in contaminated sites, monoaromatic hydrocarbons are often found as co-contaminants in petrochemical refinery and storage areas. Gasoline, for example, has benzene and toluene on $30 \%$ of its formulation. Opportunely, these compounds are also known for their potential biodegradability using aerobic microbial communities (Mathur and Majumder 2010). Aromatic hydrocarbons can be dangerous if a major exposition to humans in these environments occurs, whether in chronic or accidental releases (Hamed et al. 2003).

Recently, the detection of fluoride-based compounds has also been linked to the susceptibility of petroleum-contaminated sites to toxicological effects (Laitinen et al. 2014; Panneerselvan et al. 2016). In this context, co-contamination poses a threat for any remediation strategy of contaminated groundwater containing both hydrocarbons from refining processes and firefighting foams. Fluorinated compounds have a major role in promoting an even greater toxicity of hydrocarbons in soil. Their surfactant properties also increase the risk of spreadability of contamination plumes over time.

Bioremediation techniques are cost-effective approaches that can be applied to such contamination scenarios, by using biological agents to remove hazardous residue. Microorganism-based strategies result in the mineralization of the pollutant via microbial biodegradation processes, yielding carbon dioxide and water (Alexander 1975; Kolvenbach et al. 2014; Khatoon et al. 2017; Kapellos 2017). The biomass can either be solely composed of indigenous microorganisms from the site itself or mixed with enhanced cultures. Bioremediation aims to exploit many microbial-specific oxidation-reduction reactions, in which hydrocarbon is optimally oxidized and the most appropriated electron acceptors are selected (Martínková et al. 2009; McGenity 2014; Ghattas et al. 2017). Under the appropriate environmental conditions, benzene and toluene compounds can be co-remediated in the presence of perfluorinated compounds, thus simultaneously outputting maximum defluorination and hydrocarbon mineralization.

It is unknown if perfluorinated-adapted microbial populations are active and fully able to diverge the biotransformation process of monoaromatic hydrocarbons. A complete knowledge of metabolic pathways of hydrocarbon-degrading and fluorotelomertransforming microorganisms would allow the design of effective remediation systems specifically aimed towards both hydrocarbon mineralization and defluorination at foam-impacted sites.

In this context, our research simulated a co-contamination scenario with perfluorinated compounds, benzene, and toluene. We aimed to measure the biodegradation process in a cocontamination scenario with monoaromatic hydrocarbons commonly found in fuels (benzene, toluene) and fluorotelomers. The $\mathrm{CO}_{2}$ production rates were evaluated by a factorial design taking into account the effect of seasonality at in situ natural attenuation processes. Furthermore, aromatic hydrocarbon concentration was simultaneously monitored at a set temperature to determine the biodegradation rate of benzene and toluene in such cocontamination environment. After the biodegradation process of both benzene and toluene substrates was over, we searched for individual metabolites from the biodegradation process. Our approach complimentarily provided new insights towards the behavior of firefighting foams when released in a soil matrix.

\section{Methods}

The biodegradation was monitored using headspace analysis by gas chromatography with a thermal conductivity detector (GC-TCD) to detect $\mathrm{CO}_{2}$ production, whereas aromatics were analyzed by gas chromatography coupled to mass spectrometry (GC-MS). With such methodologies, we inferred how benzene and toluene biodegradation was influenced by the perfluorinated substances from firefighting foams.

\section{Sample collection}

Soil samples were collected in 2017 from Replan Petrobras oil

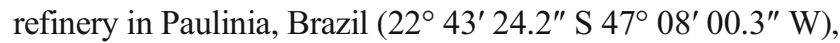
in an area close to fuel tanks. The soil was collected at around 
$0.9 \mathrm{~m}$ subsurface depth. All samples were stored in nonfluoride-based containers at $-80{ }^{\circ} \mathrm{C}$ before the assay setup. This soil sample has been chosen due to the local microbiota adaptations to biodegrade hydrocarbons. Besides, this soil has a history of the occasional release of perfluorinated compounds during firefighting training. Over 20 years ago, approximately $36,800 \mathrm{~L}$ of firefighting foam was released into this soil, when firefighters struggled to extinguish more than $5,000,000$ gal of burning diesel and gasoline. Traces of perfluorinated substances are still detected in this region nowadays (Figueredo and Sabadini 2003).

\section{Serum bottle assays}

The experiments were set up using 100-mL serum bottles. Each glass bottle contained a simulated microenvironment with soil microbiota and contaminants. In order to better evaluate environmental parameters towards benzene and toluene biodegradation, the experimental design followed a function of factors. Two factors or parameters such as temperature and perfluorinated compound concentration were chosen as the variables at three defined levels diverging from a central point $(-1,0,+1)$. Degradation rates were determined according to $\mathrm{CO}_{2}$ production in the respirometric assays. The simulated seasonality was achieved by storing our assays at different temperatures according to Table 1 .

All assays were assembled in a sterile environment. The temperature range $\left(20\right.$ to $35^{\circ} \mathrm{C}$ ) was chosen due to the temperature

Table 1 Experimental design with temperature, benzene, toluene, and fluorotelomer concentration factors

\begin{tabular}{llll}
\hline $\begin{array}{l}\text { Assay } \\
\text { number }\end{array}$ & $\begin{array}{l}\text { Factor A: } \\
\text { Temperature } \\
\left({ }^{\circ} \mathrm{C}\right)\end{array}$ & $\begin{array}{l}\text { Factor B: } \\
\text { Benzene } \\
\text { and toluene } \\
(\mu \mathrm{L})\end{array}$ & $\begin{array}{l}\text { Factor C: } \\
\text { Fluorotelomer } \\
(\mu \mathrm{L})\end{array}$ \\
\hline 1 & 35.0 & 15.0 & 10.0 \\
2 & 27.5 & 5.0 & 70.0 \\
3 & 27.5 & 15.0 & 30.0 \\
4 & 20.0 & 35.0 & 30.0 \\
5 & 27.5 & 35.0 & 30.0 \\
6 & 27.5 & 35.0 & 10.0 \\
7 & 20.0 & 15.0 & 10.0 \\
8 & 20.0 & 5.0 & 30.0 \\
9 & 35.0 & 5.0 & 30.0 \\
10 & 35.0 & 35.0 & 30.0 \\
11 & 27.5 & 15.0 & 30.0 \\
12 & 27.5 & 15.0 & 30.0 \\
13 & 20.0 & 15.0 & 70.0 \\
14 & 35.0 & 15.0 & 70.0 \\
15 & 27.5 & 5.0 & 10.0 \\
\hline
\end{tabular}

history from winter to summer from where the soil samples were collected. Both fluorotelomer and aromatic concentrations were determined from the average concentration of such contaminants in polluted sites. All these assays were monitored using our respirometric method with GC-TCD. However, the midrange temperature assays were the only ones selected for a thorough analysis of benzene and toluene consumption and biodegradation metabolite evaluation using GC-MS.

The mid-range assay quantities are specified in Table 2. The proposed soil-free medium controls $\left(C_{M}\right)$ assessed the potential impacts of media components onto fluorinated compounds. Another set of sterile controls $\left(\mathrm{C}_{\mathrm{N}}\right)$ was prepared by three consecutive soil autoclaving procedures followed by overnight freezing at $-20{ }^{\circ} \mathrm{C}$. Full assays $(\mathrm{BT}+)$ and fluoride-free assays (BT-) were amended with BT. The assays were not supplemented with antibiotic solution, and our sterile conditions were ensured with the $\mathrm{C}_{\mathrm{N}}$ only. These controls were all established to verify any losses of the compounds due to sampling procedures or inadequate sealing.

Each assay from Table 1 incubated at the lowest and highest temperature assays also had their respective controls $\left(\mathrm{C}_{\mathrm{M}}, \mathrm{C}_{\mathrm{F}}\right.$, and $C_{N}$ ), but they were omitted in Table 2 to avoid redundancy. Ethyl-benzene and xylene, which are also monoaromatic hydrocarbons as well as components of BTEX mixtures (benzene, toluene, ethyl-benzene, and xylenes), were not used in our experimental setup. This deliberate design choice was based on pilot data containing all BTEX (benzene, toluene, ethylbenzene, and xylene) components, where the consumption rates of ethyl-benzene and xylenes were all proportional. Besides, we aimed to simplify our approach, since the combined metabolic pathways involved in the degradation of such compounds can lead to even more variables that could induce erroneous conclusions. Therefore, benzene and toluene were selected as good indicators towards the whole mixture biodegradability. We will further refer to the benzene and toluene mixture as BT in this paper.

Our experiments were designed to isolate the effects of perfluorinated compounds; however, the full formulation of firefighting foams contains many other substances that could act as substrates that deviate the microbial metabolism from the targeted biodegradation process. Such substances include surfactants, diethylene glycol butyl ether, preservatives, and other non-fluorinated compounds. Along with diethylene glycol butyl ether, these substances are most of firefighting foam formulation. It is important to notice that diethylene glycol butyl ether contributes to over $80 \%$ of the total organic carbon of foams. Therefore, we used only fluorotelomers in our simulated contamination, as they were provided separately from the mixture by the local firefighting foam manufacturer. The perfluorinated portion from the commercially available $6 \%$ Sintex AFFF formulation (S1371/11, manufactured in Vinhedo, SP, Brazil) contained fluorotelomer thioether amido sulfonates (FtTAoS) ranging from 4 to 8 fluoride-bound carbon chains. 
Table 2 Biodegradation assay contents

\begin{tabular}{lllllll}
\hline Assay ID & Description & Media $(\mathrm{mL})$ & Soil $(\mathrm{g})$ & Fluorotelomer $(\mu \mathrm{L})$ & Benzene $(\mu \mathrm{L})$ & Toluene $(\mu \mathrm{L})$ \\
\hline $\mathrm{C}_{\mathrm{M}}$ & Media control & 25 & 2.5 & - & - & - \\
$\mathrm{C}_{\mathrm{F}}$ & Fluoride & 25 & 2.5 & 30 & - & - \\
$\quad \quad$ control & & & & 15 & 15 \\
$\mathrm{C}_{\mathrm{N}}$ & Sterile control & 25 & $2.5^{*}$ & 30 & 15 & 15 \\
$\mathrm{BT}-$ & No fluoride & 25 & 2.5 & - & 15 & 15 \\
$\mathrm{BT}+$ & Full assay & 25 & 2.5 & 30 & & \\
\hline
\end{tabular}

*Autoclaved soil
The minimum saline media used in our experiment proposed by Bushnell Haas (BH media) were composed of $0.2 \mathrm{~g} \mathrm{~L}^{-1} \mathrm{MgSO}_{4}, 0.02 \mathrm{~g} \mathrm{~L}^{-1} \mathrm{CaCl}_{2}, 1.0 \mathrm{~g} \mathrm{~L}^{-1} \mathrm{KH}_{2} \mathrm{PO}_{4}$, $1.0 \mathrm{~g} \mathrm{~L}^{-1} \mathrm{~K}_{2} \mathrm{HPO}_{4}, 1.0 \mathrm{~g} \mathrm{~L}^{-1} \mathrm{NH}_{4} \mathrm{NO}_{3}$, and $0.05 \mathrm{~g} \mathrm{~L}^{-1}$ $\mathrm{FeCl}_{3}$. The minimum media ensured no other substrate besides the BT and fluorotelomer would interfere with the biodegradation process. The soil itself carried organic matter into our experiments; however, it was part of our experimental design aiming to simulate a soil-microenvironment.

All vials were sealed using fluoride-free rubber stoppers. This issue was addressed to avoid any other halogenated source during experiments. Each cap was checked for their capacity of BT volatilization prevention. The materials were verified by pilot assays and controls, showing no adsorption of volatile compounds at the maximum expected duration of the experiments. Moreover, the BT mixture, with $15.0 \mathrm{mg} \mathrm{L}^{-1}$ of each compound, had its final concentration calculated according to Henry's law. We took into account the volatility of the BT compounds and left them to be stabilized for $24 \mathrm{~h}$ before injection into each vial.

The experiment lasted for 50 days, during which all vials had their headspace aliquoted for GC analysis. Simultaneously with each sampling procedure, $\mathrm{O}_{2}$ was replenished to sustain aerobiotic conditions. All experiments (Table 1) were run in triplicate and shaken at $120 \mathrm{rpm}$ for each temperature in separate incubators. The headspace was sampled every 5 days with sterile syringes and immediately analyzed. No other co-factors were added to the culture media.

\section{Respirometry using GC-TCD}

Respiration activity from the soil microcosm was monitored from headspace samples at an Agilent 6850B model GCTCD. This equipment was operated with helium as carrier gas set to a $46.7 \mathrm{~mL} \mathrm{~min}{ }^{-1}$ flow. The injection temperature was $280{ }^{\circ} \mathrm{C}$. The oven temperature was $60{ }^{\circ} \mathrm{C}$ (isothermally operated) and the detector temperature was set to $250^{\circ} \mathrm{C}$. A HP-Plot capillary column $(30 \mathrm{~m} \times 530 \mathrm{~mm} \times 40 \mathrm{~mm})$ column was used in the system. The volumes of $\mathrm{CO}_{2}$ produced were normalized to standard temperature and pressure conditions (273 K and $101.3 \mathrm{kPa})$.
Each measurement removed $250 \mu \mathrm{L}$ of headspace volume from the flasks. The same amount of compressed sterile air was added to keep pressure equilibrium between the flasks and the atmosphere, as well as sustain between 15 and $25 \%(v / v)$ of $\mathrm{O}_{2}$ throughout the entire experiment. No depressurizing was necessary for the bottles, as sterile needles inserted in the buffer valve would still result in contamination. The $\mathrm{CO}_{2}$ production rate for every 5 days was then used to produce a surface response according to the proposed factorial design.

\section{Headspace analysis of aromatic hydrocarbon using GC-MS}

We monitored the biodegradation of BT using a Shimadzu QP2010 Ultra GC-MS. The concentration of BT was monitored through a $100 \mu \mathrm{L}$ headspace aliquot injected into a Rtx$5 \mathrm{MS}$ chromatographic column. The column's stationary phase was composed of 5\% phenyl and $95 \%$ dimethylpolysiloxane $(30 \mathrm{~m} \times 0.25 \mathrm{~mm} \times 0.25 \mathrm{~mm})$. Sampling was performed every 5 days, which is also the same interval in which $\mathrm{CO}_{2}$ analyses were performed.

The parameters were modified from the method 12-154 Shimadzu for general hydrocarbon detection. The carrier gas was helium. For a reliable analysis that did not impair the equipment and the column, the injector and detector temperatures were set to $290{ }^{\circ} \mathrm{C}$, although the original methodology recommends $310^{\circ} \mathrm{C}$. Our results show no noticeable difference in signal intensity when the temperature is lowered. The BT chromatogram (Fig. 1) showed sufficient resolution to quantify the consumption of BT with an initial signal intensity greater than $5000 \mathrm{pA}$. The following temperature program was applied: 35 to $85^{\circ} \mathrm{C}$, with a $60{ }^{\circ} \mathrm{C} \mathrm{min}{ }^{-1} \mathrm{ramp}$ and then an increase from 80 to $115^{\circ} \mathrm{C}$ at a rate of $35^{\circ} \mathrm{C} \mathrm{min}^{-1}$. The total run time for each sample was $5.6 \mathrm{~min}$.

Similarly to the $\mathrm{CO}_{2}$ production rate measurements, the gas samples did not undergo any type of pre-preparation. Headspace was injected directly after removal from serum bottles. The gas withdrawal was performed with a glass gastight syringe that perforated directly through the rubber caps. Serum bottles were not opened throughout the experiment, to avoid risking any interference with the biodegradation process. 
Fig. 1 Chromatogram from a benzene (peak A) and toluene (peak B) mixture

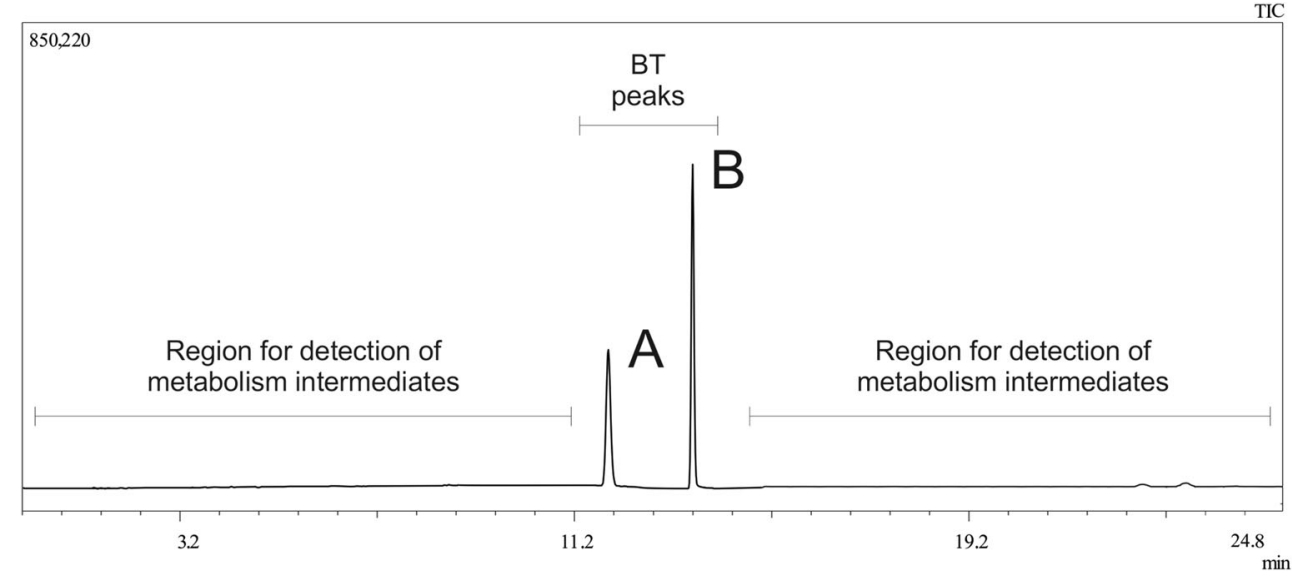

\section{Liquid extraction and analysis of biodegradation products}

After 50 days, the serum bottles were opened and mixed with $20 \mathrm{~mL}$ of ethyl acetate and $10 \mathrm{~mL}$ of dichloromethane. This solvent choice was aimed at increasing the miscibility between the solvent and the sample during the chromatographic stages. The following steps were modified from Gołębiowski et al. (2011) and Yang et al. (2017). Our method aimed towards full optimization of the extraction methodology applied to aromatic hydrocarbons. This method also ensured that most of the metabolites that eventually formed inside the flasks would be extracted as well. The 1:1:2 mixture of solvent and sample was subjected to manual stirring for $5 \mathrm{~min}$ and sonicated for $30 \mathrm{~min}$. In addition, the soil particles were separated from the liquid phase by centrifugation at $15,000 \mathrm{~g}$ for $15 \mathrm{~min}$ prior to further processing. The contents were rapidly transferred into $2.5-\mathrm{mL}$ vials and analyzed by $\mathrm{GC}-\mathrm{MS}$.

Our method for BT quantification from headspace aliquots was extremely optimized for such peaks. However, such short running time was not adequate for the detection of metabolic by-products which may have been formed during the BT biodegradation process. For this reason, we expanded this method for the detection of compounds over a wider spectrum of chromatographic separation. Figure 1 shows the regions in which the presence of aromatic biodegradation intermediates was expected in this expanded method. The products could be smaller biodegraded compounds or even larger metabolic intermediates throughout the process.

The parameters we used were similar to the headspace analysis, except that temperature program applied was $35^{\circ} \mathrm{C}$ kept for $5 \mathrm{~min}$, then a ramp of $10^{\circ} \mathrm{C} \mathrm{min}{ }^{-1}$ until $220^{\circ} \mathrm{C}$ was reached followed by a steeper temperature ramp at $20{ }^{\circ} \mathrm{C} \min ^{-1}$ until $250{ }^{\circ} \mathrm{C}$ was reached. The total run time for each sample was $25.0 \mathrm{~min}$.

Mass spectrum $(70 \mathrm{eV})$ was specifically used to identify the new compounds. While the BT biodegradation in the previous section was detected from peak area integration and a standard solution-based calibration curve, the mass spectrometry allowed the identification of new compounds formed after 50 days of biodegradation. The compounds were analyzed according to their mass spectra. The ion source of the massspectrometer was kept at $200{ }^{\circ} \mathrm{C}$. A detector containing an electron ionization source and a quadrupole mass analyzer was operated in scan mode for chemical identification. The detector was at $250{ }^{\circ} \mathrm{C}$ and the source of ions at $200^{\circ} \mathrm{C}$. The NIST11 Mass Spectrum Library was used for identification of biodegradation intermediates.

\section{Results and discussion}

\section{$\mathrm{CO}_{2}$ production rate}

The interaction effects of temperature, BT, and fluorotelomer concentration on the degradation rates have been illustrated with the contour plots shown in Fig. 2.

The contour plots in Fig. 2a depict that the degradation rate increases at higher temperatures. The observed temperature effect on $\mathrm{CO}_{2}$ production rate was expected since higher temperatures are related to an increase in microbial activity. Seasonality has a detectable effect during summer $\left(35^{\circ} \mathrm{C}\right)$ and winter $\left(20^{\circ} \mathrm{C}\right)$ conditions, yielding different $\mathrm{CO}_{2}$ production rates. In other words, GC-TCD respirometry was a very sensitive technique to detect variations in $\mathrm{CO}_{2}$ concentrations within mild weather conditions. Such conditions, however, were observable in a controlled laboratory environment. Extreme temperatures would be beyond the resolution limit of our method, as column saturation would be too high in summer or compound degradation would be too low to be detected in winter.

No isolated peaks were observed at the response surface for both temperature and fluorotelomer concentration. The contour plots show that there is a semi-linear approach to $\mathrm{CO}_{2}$ production response when all three factors are considered. Even though there is no peak for specific concentrations or 


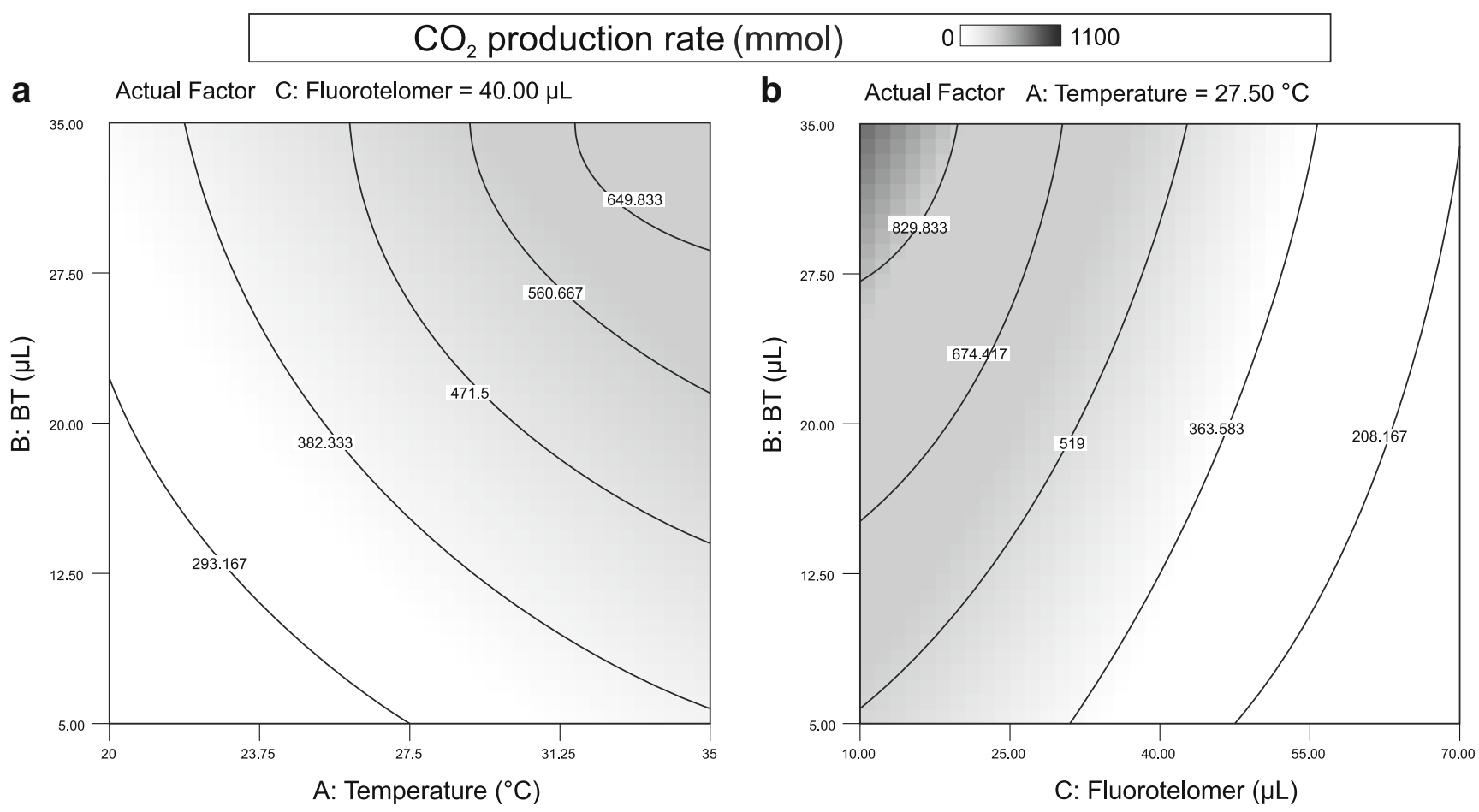

Fig. 2 Contour plot of temperature, benzene, toluene, and fluorotelomer concentrations factors in $\mathrm{CO}_{2}$ production rate response at a set fluorotelomer concentration (a) and temperature (b)

temperatures, the tested parameters (temperature, BT, and fluorotelomer) were all related to $\mathrm{CO}_{2}$ variations.

Higher temperatures increased $\mathrm{CO}_{2}$ production rate, while higher concentrations of fluorotelomer inhibited the biodegradation process. The BT factor, however, caused a directly proportional increase in $\mathrm{CO}_{2}$ production at higher concentrations. Increased benzene and toluene concentration turned them into a readily available substrate to the indigenous microorganisms in our soil samples, as a result of increased degradation rates. It is worth mentioning, however, that the BT concentrations in this study were carefully planned to be below toxicity levels, since beyond $200 \mathrm{mg} \mathrm{L}^{-1}$ the $\mathrm{CO}_{2}$ response was expected to decrease, thus indicating inhibition due to the toxicity of benzene. The inhibition effect of toluene was also reported at a concentration as low as $158 \mathrm{mg} \mathrm{L}^{-1}$, according to Monero et al. (2003).

The response surface contours (Fig. 2) can also properly identify the interactions between variables and predict the maximum response of a given measured parameter. According to Zhao et al. (2016), a circular contour pattern is likely to have better interaction between the variables, whereas an elliptical pattern indicates non-significant interaction. The contour plots observed in our co-contaminations containing fluorotelomer and BT were all circular for the fluorotelomer factor (Fig. 2b), indicating a relevant interaction between the factors.

The respirometric analysis pointed towards the inhibition of $\mathrm{CO}_{2}$ production when higher concentrations of fluorotelomer are present. There is a balance between an increase in $\mathrm{CO}_{2}$ production due to the presence of higher concentrations of
BT compounds and an inhibition of microbial activity at higher firefighting foam concentrations. Contrary to BT, the high concentration of fluorotelomers in our assays led to toxicological effects that may have halted several cellular functions and therefore lowered $\mathrm{CO}_{2}$ production to a minimum.

Our mixed-substrate assays simulated the main bioremediation challenge when creating new cost-effective and viable technologies for environmental remediation applications (Vinuselvi et al. 2012; Aparício et al. 2018). We encourage further studies that promote an in-depth investigation of the effects of perfluorinated compounds in diminishing removal efficiency of aromatics. This is particularly urgent since environmental contamination containing both compounds is fairly common in the petrochemical industry. Understanding the BT biodegradation profile may also provide additional information, compared to $\mathrm{CO}_{2}$ monitoring. Therefore, $\mathrm{BT}$ consumption profile can elucidate the key role of fluorotelomer in the biodegradation processes.

\section{Benzene and toluene biodegradation}

The difference between datasets from BT assays was considered statistically significant $(p>0.05)$ compared to controls $\left(\mathrm{C}_{\mathrm{M}}, \mathrm{C}_{\mathrm{N}}\right.$, and $\left.\mathrm{C}_{\mathrm{S}}\right)$. In fact, control assays did not statistically vary when compared to each other, with threshold variations in soil controls $\left(\mathrm{C}_{\mathrm{S}}\right)$. The biodegradation of the individual BT components throughout 50 days is shown in Fig. 3.

The soilless assays $\left(\mathrm{C}_{\mathrm{M}}\right)$ yielded null microbial activity (as expected from non-inoculated media). No aromatic 
Fig. 3 Benzene (a) and toluene (b) concentration in the biodegradation assays
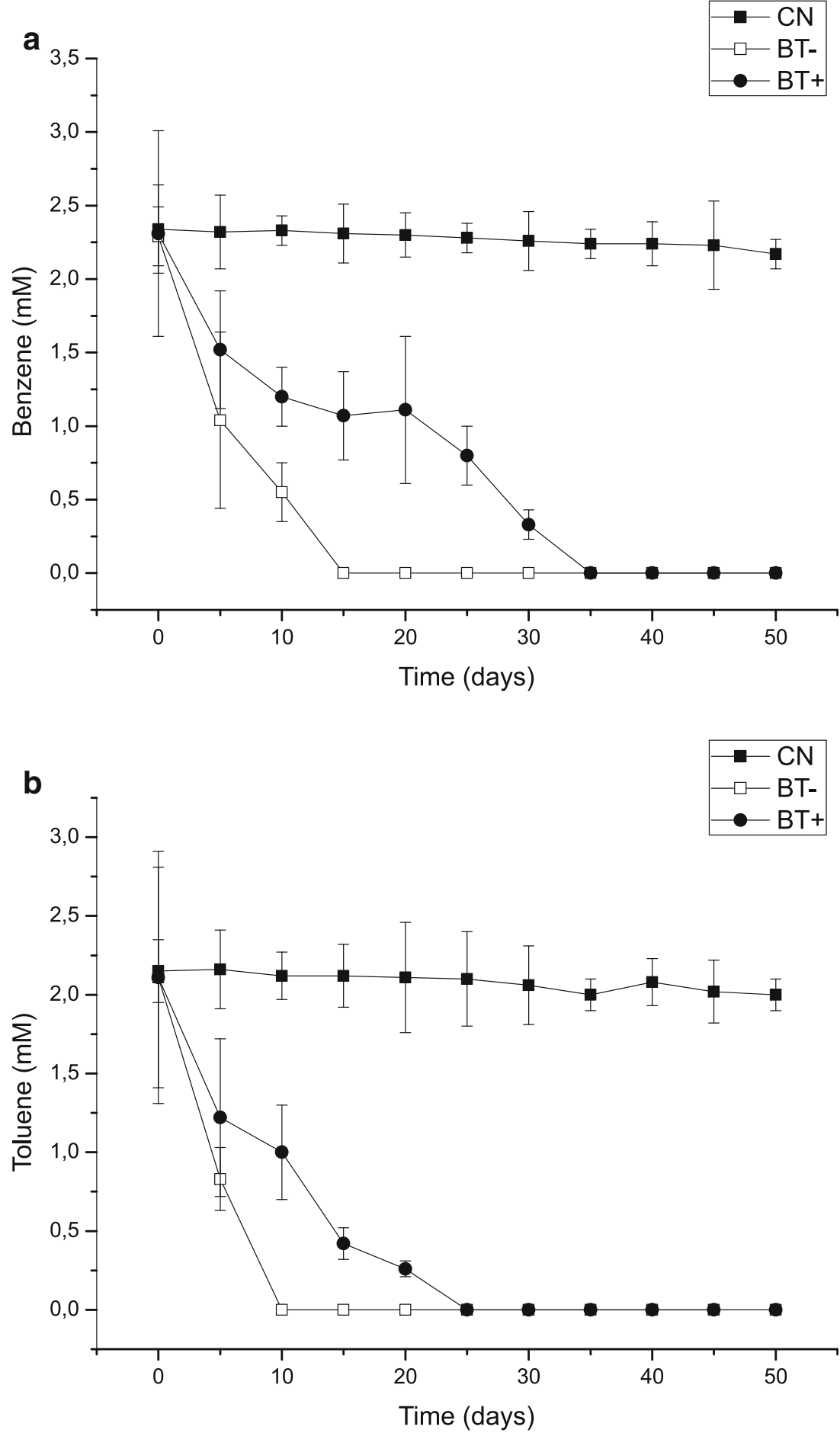

biodegradation was detected within those assays. This is important since the minimum output confirmed that no leaks or adsorption of volatiles occurred during the course of our entire experiment. Even though benzene and toluene substrates were depleted at less than 40 days for each assay, we kept the assays incubated for 50 days, since the biodegradation process of aromatics could still be occurring.
Figure 3 shows the effect of mid-range fluorotelomer concentration on BT consumption. Based on our results, benzene and toluene did not benefit from the addition of perfluorinated substances in culture media. Without fluorotelomer, the biodegradation of each BT component was seen to occur at a much higher rate than that of other assays. The absence of an adaption phase to the BT substrate during consumption form BT- assays means that no sudden inhibition to culture 
growth was caused by benzene or toluene. This might be due to the proper enrichment at a tolerable BT concentration (Kim et al. 2005; Khodaei et al. 2017).

On average, benzene and toluene were depleted 17.5 days earlier in BT- assays than those in BT+ assays. We believe that this is not due to a preferential consumption of fluorotelomers over BT by the indigenous microbes, whose metabolic pathways will less likely prioritize perfluorinated compounds as substrate. There was a noticeable decrease in degradation rates of $\mathrm{BT}$ when perfluorinated compounds were present. This decrease signifies that the fluorotelomer repressed BT catabolism in batch cultures. Thus, fluorotelomers were a dominant repressing substrate for our indigenous soil microbiota from a petrochemical plant. We hypothesize that BT consumption was found to be reduced in the presence of fluorotelomers due to their toxicity to the indigenous soil microbiota, since monoaromatic consumption rate decreased only when fluorotelomers were present in the batches, hence indicating inhibition.

From Fig. 3, it can also be concluded that monoaromatic uptake by the soil inoculum had undergone some preference mechanism. In other words, one of the aromatics was preferably consumed as a carbon source among the two available hydrocarbons. Toluene removal efficiency, in particular, was notably higher than benzene. The results on toluene consumption were much higher compared to the ones found in a broad literature review by Yeom et al. (1997), as well as recent results by Su et al. (2014) and Rajamanickam et al. (2017). Therefore, the conditions adopted in this study demonstrated that an unusual approach (i.e., testing fluorocarbon effect in soil microbiota) found conditions that may favor an improved biodegradation process of toluene, despite the inhibition effects of perfluorinated compounds at a higher concentration.

Benzene took a longer time to be consumed than toluene. Still, both degradation pathways were activated when BT was available. On the other hand, fluorotelomer negatively affected the biodegradation rates. It is uncertain whether this also means competitive inhibition, as BT degradation pathways are too complex. Even though the analysis of metabolic pathways is beyond the scope of this study, we further investigated the formation of intermediate compounds after the proposed 50 days of biodegradation monitoring. This was the reason for incubating our assays 20 days after benzene and toluene were no longer detected at headspace sample analyses.

\section{Biodegradation intermediates formation}

After the 50-day biodegradation period, we searched for metabolites that could have been formed in the headspace or the soil matrix. The differential biodegradation profile between assays with and without fluorinated compounds prompted us to perform a detailed analysis of the chromatogram to search for intermediates of aromatic hydrocarbon biodegradation
(Fig. 4) by cross-referencing peaks with other common known BT degradation intermediates at the NIST11 Mass Spectrum Library.

In order to determine the metabolic intermediates during BT biodegradation, samples were collected from the optimized mid-range fluorotelomer assays after 50 days of incubation for analysis in GC-MS. The result of GC-MS showed the presence of various compounds. However, the compound formation was different when perfluorinated compounds were present. Many commonly reported biodegradation intermediates of aromatic hydrocarbons (Singh and Fulekar 2010) such as catechol (Fig. 4, indicated as 9), maleic acid (Fig. 4, indicated as 4), 2-hydroxymuconate semialdehyde (Fig. 4a, indicated as 7), and p-cresol (Fig. 4, indicated as 8) were found in our chromatograms.

The presence of metabolites confirms the biodegradation of benzene and toluene by the indigenous microbial culture from refinery soil. Most of the compounds were associated with the aerobic activity of the dioxygenase enzyme. The mono- and dioxygenases are often involved in the oxidization of aromatic ring of benzene and toluene by incorporating two oxygen atoms to form 2-hydroxysubstituted radicals (Littlejohns and Daugulis 2008; Kim et al. 2009). The metabolic intermediates produced during the BT biodegradation in this study have been supported by many established biodegradation pathways in literature. The microbial adaptation to aromatics (especially benzene and toluene) was broadly studied, as wisely compiled by El-Naas et al. (2014) in their review. Recent studies on aromatics suggest that the adaptation of microbial communities affects the usually reported biodegradation pathways, since co-metabolites may result in higher efficiency of aromatic removal from a contaminated site.

Even though BT+ (Fig. 4b) and BT- (Fig. 4a) showed differences in metabolites, they shared similarities to many of the expected compounds. It is worth mentioning that the first peak (Fig. 4, indicated as 1) corresponds to the elution solvent (ethyl acetate) and it is not a biodegradation metabolite. The main similarity between intermediates in BT+ and BT- assays, which is also the source of many subsequent oxidation steps, is catechol (Fig. 4, indicated as 9). Catechol was found in both of our assays (Fig. 4, indicated as 9), in which its further mineralization is associated with the support of biomass growth, despite the presence of fluorotelomers. Under certain circumstances, this compound is usually converted to 2-hydroxymuconate semialdehyde (Fig. 4a, indicated as 7), among many others, and is ultimately converted to substrates of the citric acid cycle that ultimately leads to the final biodegradation step that produces $\mathrm{CO}_{2}$ and water (Meckenstock et al. 2004; Kadri et al. 2017). The 2hydroxymuconate semialdehyde, however, was not found in BT+ assays (Fig. 4b), thus indicating a metabolic shift due to fluorotelomer influence. 

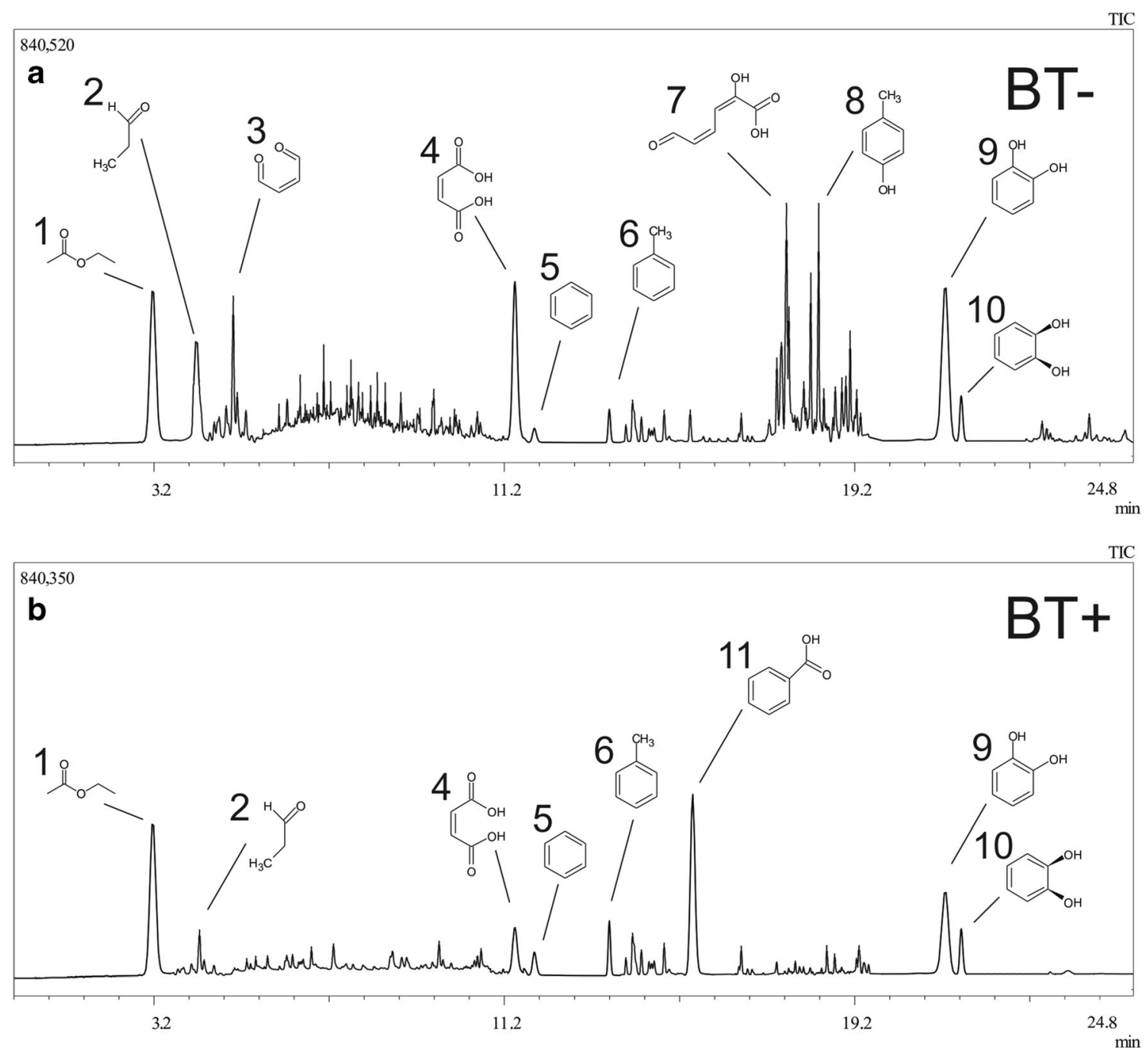

Fig. 4 Post-biodegradation chromatogram without BT (a) and with BT (b) showing peaks identified by their mass spectra indicated as 1, ethyl acetate; 2 , propionaldehyde; 3 , butanediol; 4 , maleic acid; 5 , benzene; 6 ,

As shown in Fig. 4, GC-MS analysis of BT and its byproducts (after microbial consumption) had possibly identified two exclusive metabolites via similarity search on the NIST11 library. The benzene and toluene molecules were shown as very small peaks after the biodegradation process (Fig. 4, indicated as 5 and 6 , respectively) when compared to the control chromatogram (Fig. 1). The lengthy run time with a total retention time of 24.88 min allowed a thorough capture of many compounds. An alternative intermediate peak eluted at $15.49 \mathrm{~min}$ exclusively at BT+ assays (Fig. 3b, indicated as 11) compared to the BT - chromatogram. It was identified as benzoic acid and is associated with alternate toluene biodegradation routes. Considering this molecule is a late compound in toluene $\mathrm{H}$ abstraction pathway (Ramos et al. 1997), we may conclude that biodegradation occurred up to more advanced steps. Despite the lower diversity of compounds in BT+ assays, an alternate pathway was triggered by the presence of perfluorinated toluene; 7, 2-hydroxymuconate semialdehyde; 8, p-cresol; 9, cathecol; 10, cis-1,2-dihydrobenzene-1,2-diol; 11, benzoic acid

compounds. Peak intensity was much lower in BT+ than that in BT- though, which confirms the minor biodegradation rate found in both headspace and respirometric approaches.

Our previous studies on aromatics and fluorinated compounds (Montagnolli et al. 2017) suggested that the adaptation of microbial communities to the fluorotelomers had an impact on the biotransformation of the carbon sources. The fluorinated compounds changed the BTEX biodegradation products, as the halogenated substances could have contributed to a shift in styrene concentrations in co-contamination scenarios. Catechol was also rapidly metabolized to further metabolic stages. At that time, we were unsure whether any carbon source or specific aromatic biodegradation enzymes could further expand biotransformation. These results led to the follow-up experiments proposed in this paper and the search for other compounds (such as benzoic acid and p-cresol) that could be related to alternative pathways triggered by fluorotelomers. 
While we cannot assure that our proposed method had scanned all compounds present in our post-biodegradation samples due to factors such as the volatility, melting temperature, molecular structures, and various other conditions that are important for a successful final elution, our approach was able to detect benzene and toluene metabolites. All biodegradation intermediates found in this study share properties such as high volatility, low molecular weight, and low boiling temperature. The GC-MS-based metabolite analysis had a high sensitivity and successfully confirmed BT in many established biodegradation pathways available in current literature (Bielefeldt and Stensel 1999; Gieg et al. 2014; Varjani 2017). The differences between metabolites in BT+ and BT- assays, however, were the most relevant result. The discovery of an unusual environmental behavior of co-contamination scenarios can help unraveling the effect of enzyme expression on metabolism and organism performance (Jonsson et al. 2004; Schauer et al. 2005; Vogt et al. 2016) in corresponding bioremediation strategies.

Other apparent metabolites that eluted at 5.6 and $17.9 \mathrm{~min}$ were butanediol and 2-hydroxymuconate semialdehyde, respectively (according to the NIST library). These compounds were only present in assays without perfluorinated substances. Both intermediates detected by GC-MS could be the result of specific enzymatic mechanisms that are inactivated in the presence of fluoride or enzymes in the classical pathways that are inhibited, leading to alternate pathways. It has been long demonstrated (over 20 years ago) by Yeom et al. (1997) that even microbes adapted to only one of aromatic compounds may change their enzymatic mechanisms, thus attaining the ability to degrade toluene and other monoaromatics faster than control assays through — at that time - unidentified pathways. However, there are few studies that link perfluorinated compound co-contamination to hydrocarbon biodegradation. Therefore, the differential biodegradation mechanisms observed in our co-contamination scenarios through GC-MS analysis might lead to the development of different bioremediation strategies that can predict differential accumulation of compounds in the environment.

\section{Conclusions}

Perfluorinated substances caused the biodegradation process of monoaromatic hydrocarbons to significantly differ. When seasonal effects were considered, a reduced biodegradation rate (in terms of $\mathrm{CO}_{2}$ production rate) indicated inhibitory effects, despite different temperatures. In fact, the sole presence of such organo-halogenated substances was capable of reducing the microbial uptake of benzene and toluene. Moreover, the intermediate compound analysis confirmed that a specific compound could be detected exclusively to soil microbiota contaminated with perfluorinated compounds. In summary, we found that fluorotelomers can trigger a new metabolic pathway which may lead to previously unknown biodegradation profiles and microbial behavior.

Thus, we propose that transient components of the benzene and toluene degradation may be differentially formed, causing the BT and perfluorinated co-contaminations to go through switched metabolic stages under the presence of fluoride in a co-contamination scenario. The fluorotelomers contributed to the development of different concentrations of by-products, even though no production/consumption spikes occurred when concentration and temperature factors were evaluated. The synergic effect of firefighting foams on benzene and toluene biodegradation might provide a better perspective for more efficient bioremediation techniques and fully optimized environmental cleaning-up strategies. The better understanding of microbial behavior and biodegradation patterns at firefighting sites can narrow current efforts for the improvement of ongoing development on environmental biotechnology. Therefore, we encourage further studies about novel benzene and toluene metabolites that expand the current knowledge of the effects of organo-halogenated substances during the biodegradation of monoaromatics.

Acknowledgements Our research group acknowledges CAPES (Coordenação de Aperfeiçoamento de Pessoal de Nível Superior), CNPq (Conselho Nacional de Desenvolvimento Científico e Tecnológico), FUNDUNESP (Fundação para o Desenvolvimento da UNESP), PRH-ANP/MCT (Programa de Formação de Recursos Humanos em Geologia do Petróleo e Ciências Ambientais Aplicadas ao Setor de Petróleo e Gás), and UNESP (Universidade Estadual Paulista "Julio de Mesquita Filho") for the financial support.

\section{References}

Alexander M (1975) Environmental and microbiological problems arising from recalcitrant molecules. Microb Ecol 2:17-27

Aparício JD, Raimondo EE, Gil RA, Benimeli CS, Polti MA (2018) Actinobacteria consortium as an efficient biotechnological tool for mixed polluted soil reclamation: experimental factorial design for bioremediation process optimization. J Hazard Mater 342:408-417

Bielefeldt AR, Stensel HD (1999) Evaluation of biodegradation kinetic testing methods and longterm variability in biokinetics for BTEX metabolism. Water Res 33:733-740

Borg A, Njå O (2013) The concept of validation in performance-based fire safety engineering. Saf Sci 52:57-64

Chettouh S, Hamzi R, Benaroua K (2016) Examination of fire and related accidents in Skikda Oil Refinery for the period 2002-2013. J Loss Prevent Proc 41:186-193

Choi H, Lee J (2011) Groundwater contamination and natural attenuation capacity at a petroleum spilled facility in Korea. J Environ Sci 23: $1650-1659$

Cholakov GS (2016) Air quality and the petroleum industry. In: de la Guardia M, Armenta S (eds) Comprehensive analytical chemistry, 73rd edn. Elsevier, New York, pp 563-587

Dauchy X, Boiteux V, Bach C, Rosin C, Munoz J (2017) Per- and polyfluoroalkyl substances in firefighting foam concentrates and water samples collected near sites impacted by the use of these foams. Chemosphere 183:53-61 
Donaldson D (2016) New foam improves firefighting capabilities. Mater Today 19:63

El-Naas MH, Acio JA, Telib AE (2014) Aerobic biodegradation of BTEX: progresses and prospects. J Environ Chem Eng 2:1104 1122

Fernández EL, Merlo EM, Mayor LR, Camacho JV (2016) Kinetic modelling of a diesel-polluted clayey soil bioremediation process. Sci Total Environ 557:276-284

Figueredo RCR, Sabadini E (2003) Firefighting foam stability: the effect of the drag reducer poly(ethylene) oxide. Colloids Surf A Physicochem Eng Asp 215:77-86

Freije AM (2015) Heavy metal, trace element and petroleum hydrocarbon pollution in the Arabian Gulf: review. Journal of the Association of Arab Universities for Basic and Applied Sciences 17:90-100

Ghattas A, Fischer F, Wick A, Ternes TA (2017) Anaerobic biodegradation of (emerging) organic contaminants in the aquatic environment. Water Res 116:268-295

Gieg LM, Fowler SJ, Berdugo-Clavijo C (2014) Syntrophic biodegradation of hydrocarbon contaminants. Curr Opin Biotechnol 27:21-29

Gołębiowski M, Siedlecka E, Paszkiewicz M, Brzozowski K, Stepnowski P (2011) Perfluorocarboxylic acids in cell growth media and technologically treated waters: determination with GC and GC-MS. J Pharm Biomed Anal 54:577-581

Hamed TA, Bayraktar E, Mehmeto T, Mehmeto U (2003) Substrate interactions during the biodegradation of benzene, toluene and phenol mixtures. Process Biochem 39:27-35

Hinnant KM, Giles SL, Ananth R (2017) Measuring fuel transport through fluorocarbon and fluorine-free firefighting foams. Fire Saf J 91:653-661

Jonsson P, Gullerg J, Nordstrom A, Kusano M, Kowalczylk M, Sjostrom M, Moritz T (2004) A strategy for identifying differences in large series of metabolomic samples analyzed by GC/MS. Anal Chem 76: $1738-1745$

Kadri T, Rouissi T, Brar SK, Cledon M, Sarma S, Verma M (2017) Biodegradation of polycyclic aromatic hydrocarbons (PAHs) by fungal enzymes: a review. J Environ Sci 51:52-74

Kapellos GE (2017) Microbial strategies for oil biodegradation. In: Becker SM (ed) Modeling of microscale transport in biological processes. Academic Press, New York, pp 19-39

Khatoon N, Jamal A, Ali MI (2017) Polymeric pollutant biodegradation through microbial oxidoreductase: a better strategy to safe environment. Int J Biol Macromol 105:9-16

Khodaei K, Nassery HR, Asadi MM, Mohammadzadeh H, Mahmoodlu MG (2017) BTEX biodegradation in contaminated groundwater using a novel strain (Pseudomonas sp. BTEX-30). International Biodeterioration \& Biodegradation 116:234-242

Kim SJ, Kweon O, Cerniglia CE (2009) Proteomic applications to elucidate bacterial aromatic hydrocarbon metabolic pathways. Curr Opin Microbiol 12:301-309

Kolvenbach BA, Helbling DE, Kohler HE, Corvini PF (2014) Emerging chemicals and the evolution of biodegradation capacities and pathways in bacteria. Curr Opin Biotechnol 27:8-14

Laitinen JA, Koponen J, Koikkalainen J, Kiviranta H (2014) Firefighters' exposure to perfluoroalkyl acids and 2-butoxyethanol present in firefighting foams. Toxicol Lett 231:227-232

Littlejohns JV, Daugulis AJ (2008) Kinetics and interactions of BTEX compounds during degradation by a bacterial consortium. Process Biochem 43:1068-1076

Martínková L, Uhnáková B, Pátek M, Nešvera J, Kren V (2009) Biodegradation potential of the genus. Environ Int 35:162-177

Mathur AK, Majumder CB (2010) Kinetics modelling of the biodegradation of benzene, toluene and phenol as single substrate and mixed substrate by using Pseudomonas putida. Chem Biochem Eng Q 24: 101-109

McGenity TJ (2014) Hydrocarbon biodegradation in intertidal wetland sediments. Curr Opin Biotechnol 27:46-54
Meckenstock RU, Safinowski M, Griebler C (2004) Anaerobic degradation of polycyclic aromatic hydrocarbons. FEMS Microbiol Ecol 49: $27-36$

Meyer DD, Beker SA, Bücker F, Peralba MCR, Frazzon APG, Osti JF, Andreazza R, Camargo FAP, Bento FM (2014) Bioremediation strategies for diesel and biodiesel in oxisol from southern Brazil. Int Biodeterior Biodegradation 95:356-363

Mishra N, Ayoko GA, Morawska L (2016) Atmospheric polycyclic aromatic hydrocarbons in the urban environment: occurrence, toxicity and source apportionment. Environ Pollut 208:110-117

Monero A, Lanza L, Zilli M, Sene L, Converti A (2003) Batch kinetics of Pseudomonas sp. growth on benzene. Modeling of product and substrate inhibitions. Biotechnol Prog 19:676-679

Montagnolli RN, Lopes PRM, Bidoia ED (2014) Screening the toxicity and biodegradability of petroleum hydrocarbons by a rapid colorimetric method. Arch Environ Contam Toxicol 12:1-12

Montagnolli RN, Lopes PRM, Cruz JM, Claro MT, Quiterio GM, Bidoia ED (2017) Metabolical shifts towards alternative BTEX biodegradation intermediates induced by perfluorinated compounds in firefighting foams. Chemosphere 173:49-60

Nadim F, Hoag GE, Liu S, Carley RJ, Zack P (1999) Detection and remediation of soil and aquifer systems contaminated with petroleum products: an overview. J Pet Sci Eng 26:169-178

NFPA - National Fire Protection Association (1991) Fire protection handbook, 17th edn. NFPA, Quincy

Nolan DP (2011) Historical survey of fire and explosions in the hydrocarbon industries. In: Nolan DP (ed) Handbook of fire and explosion protection engineering principles, 2nd edn. William Andrew Publishing, Oxford, pp 71-82

Oberoi AS, Philip L (2017) Variation in toxicity during the biodegradation of various heterocyclic and homocyclic aromatic hydrocarbons in single and multi-substrate systems. Ecotoxicol Environ Saf 135: $337-346$

Panneerselvan P, Sivaram AK, Mallavarapu M, Naidu R (2016) Evaluation of cyto- and genotoxic effects of Class B firefighting foam products: Tridol-S 3\% AFFF and Tridol-S 6\% AFFF to Allium cepa. Environmental Technology \& Innovation 6:185-194

Postigo C, Martinez DE, Grondona S, Miglioranza KSB (2017) Groundwater pollution: sources, mechanisms, and prevention. In: Elias SA (ed) Reference module in earth systems and environmental sciences. Elsevier, New York, pp 143-162

Qin XS, Huang GH, He L (2009) Simulation and optimization technologies for petroleum waste management and remediation process control. J Environ Manag 90:54-76

Rajamanickam R, Kaliyamoorthi K, Ramachandran N, Baskaran D, Krishnan J (2017) Batch biodegradation of toluene by mixed microbial consortia and its kinetics. International Biodeterioration \& Biodegradation 119:282-288

Ramos JL, Marques S, Timmis KN (1997) Transcriptional control of the Pseudomonas TOL plasmid catabolic operons is achieved through an interplay of host factors and plasmid-encoded regulators. Annu Rev Microbiol 51:341-372

Runefors M, Johansson N, van Hees P (2017) The effectiveness of specific fire prevention measures for different population groups. Fire Saf J 91:1044-1050

Singh D, Fulekar MH (2010) Benzene bioremediation using cow dung microflora in two phase partitioning bioreactor. J Hazard Mater 175: 336-343

Su Y, Zhang X, Wei X, Kong J, Xia F, Li W, He R (2014) Evaluation of simultaneous biodegradation of methane and toluene in landfill covers. J Hazard Mater 274:367-375

Tobiszewski M, Namieśnik J (2012) PAH diagnostic ratios for the identification of pollution emission sources. Environ Pollut 162:110-119

Varjani SJ (2017) Microbial degradation of petroleum hydrocarbons. Bioresour Technol 223:277-286 
Vecitis CD, Park H, Cheng J, Mader BT, Hoffmann MR (2009) Treatment technologies for aqueous perfluorooctanesulfonate (PFOS) and perfluorooctanoate (PFOA). Front Environ Sci En 3:129-151

Vinuselvi P, Kim MK, Lee SK, Ghim CM (2012) Rewiring carbon catabolite repression for microbial cell factory. BMB Rep 45:59-70

Vogt C, Dorer C, Musat F, Richnow H (2016) Multi-element isotope fractionation concepts to characterize the biodegradation of hydrocarbons - from enzymes to the environment. Curr Opin Biotechnol 41:90-98

Wang S, Kuo Y, Hong A, Chang Y, Kao C (2016) Bioremediation of diesel and lubricant oil-contaminated soils using enhanced landfarming system. Chemosphere 164:558-567

Yang Y, Li J, Xi B, Wang Y, Tang J, Wang Y, Zhao C (2017) Modeling BTEX migration with soil vapor extraction remediation under lowtemperature conditions. J Environ Manag 203:114-122
Yeom SH, Yoo YJ, Lee JW (1997) The importance of microbial adaptation in the degradation of BTX - studies in environmental science, 1 st edn. Elsevier, New York

Zhang X, Mehaffey J, Hadjisophocleous G (2016) Life risks due to fire in mid- and high-rise, combustible and non-combustible residential buildings. J Build Eng 8:189-197

Zhao X, Wang L, Ma F, Bai S, Yang J, Qi S (2016) Pseudomonas sp. ZXY-1, a newly isolated and highly efficient atrazine-degrading bacterium, and optimization of biodegradation using response surface methodology. J Environ Sci 54:152-159

Zhuo S, Du W, Shen G, Wang R, Pan X, Li T, Han Y, Li Y, Pan B, Peng X, Cheng H, Wang X, Shi G, Xing B, Tao S (2017) Urban air pollution and health risks of parent and nitrated polycyclic aromatic hydrocarbons in two megacities, southwest China. Atmos Environ $166: 441-453$ 